\title{
MicroRNA-4722-5p and microRNA-615-3p serve as potential biomarkers for Alzheimer's disease
}

\author{
YAN LIU, YUHAO XU and MING YU \\ Department of Neurology, The Affiliated Hospital of Jiangsu University, Zhenjiang, Jiangsu 212001, P.R. China
}

Received August 17, 2021; Accepted November 9, 2021

DOI: $10.3892 / \mathrm{etm} .2022 .11166$

\begin{abstract}
The aim of the present study was to investigate the expression levels of microRNA(miR)-4722-5p and miR-615-3p in Alzheimer's disease (AD) and their diagnostic value. Blood samples were collected from 33 patients with AD and 33 healthy controls, and an $\beta$-amyloid (A $\beta) 25$-35-induced PC12 cell model was also established. The relative mRNA expression levels of miR-4722-5p and miR-615-3p were detected using reverse transcription-quantitative PCR. The correlations between the mRNA expression levels of the two miRNAs and the mini-mental state examination (MMSE) scores were analyzed, and the receiver operating characteristic curve was used to assess the diagnostic value of miR-4722-5p and miR-615-3p in AD. Functional enrichment analysis of the miRNA target genes was performed using The Database for Annotation, Visualization and Integrated Discovery database and the $\mathrm{R}$ language analysis package. The mRNA expression levels of miR-4722-5p and miR-615-3p were increased in patients with AD and the A $\beta 25-35$-induced PC12 cell model. The mRNA expression levels of miR-4722-5p and miR-615-3p were negatively correlated with MMSE scores, and the combination of the two miRNAs for AD had an improved diagnostic value than that of each miRNA alone. The results of Gene Ontology (GO) enrichment analysis showed that the target genes of miR-4722-5p were found in the cytoplasm and cytosol, and were mainly involved in protein folding and cell division. The molecular functions included protein binding and GTPase activator activity. The results of Kyoto Encyclopedia of Genes and Genomes analysis showed that miR-4722-5p was associated with the regulation of dopaminergic synapses and mTOR signaling pathways. GO enrichment analysis also revealed that the target genes of miR-615-3p were located in the nucleus and cytoplasm, were involved in the regulation of transcription and protein phosphorylation, and were associated with protein
\end{abstract}

Correspondence to: Professor Ming Yu, Department of Neurology, The Affiliated Hospital of Jiangsu University, 438 Jiefang Road, Zhenjiang, Jiangsu 212001, P.R. China

E-mail: yuming7251@163.com

Key words: Alzheimer's disease, serum, microRNAs, diagnosis, PC12 cell line binding, metal ion binding and transcription factor activity. The target genes of miR-615-3p played important roles in the regulation of the Ras and FoxO signaling pathways. In conclusion, miR-4722-5p and miR-615-3p may be potential biomarkers in the early diagnosis of AD.

\section{Introduction}

Dementia is a syndrome that is clinically characterized by progressive loss of intelligence and is accompanied by different degrees of personality changes. Alzheimer's disease (AD) is the most common cause of dementia, accounting for $60-80 \%$ of all types of dementia (1). AD is divided into two types based on the age of onset: Early-onset AD (age, $<65$ years) and late-onset $\mathrm{AD}$ (age, $>65$ years) (2). $\beta$-amyloid $(\mathrm{A} \beta$ ) deposition and tau protein hyperphosphorylation comprise two major neuropathological features of AD (3). To date, the etiology of $\mathrm{AD}$ is unknown and may be associated with the interactions of the environment, genetics and other factors (4). For example, results of a previous study demonstrated that Ginkgo biloba extract exhibited neuroprotective antioxidant effects in rat models of AD (5). Moreover, variants of presenilin (PSEN), including PSEN1 and PSEN2, are associated risk factors of $\mathrm{AD}$ (6). Therefore, studies on $\mathrm{AD}$ biomarkers have become a hot topic in recent years. It has been shown that increased levels of neurofilament and neurogranin in the cerebrospinal fluid of patients with AD had the potential to predict the progression of AD (7). It has also been found that an increase or decrease in the expression levels of FOXO3A, lysosomal proteins and flotillin in the serum may act as novel diagnostic markers of AD (8-10). At present, only five drugs have been approved for the treatment of $\mathrm{AD}$, and these drugs can only improve the clinical symptoms in patients with AD and not delay the progression of the disease $(11,12)$. Therefore, identifying effective biomarkers is essential for early clinical diagnosis and guidance of drug therapy for patients with AD.

MicroRNAs (miRNA/miR) are a class of endogenous small non-coding RNAs. The function of miRNAs is to regulate gene expression by inhibiting the translation or promoting the degradation of target mRNAs (13). There are abundant and stable miRNAs in the blood, cerebrospinal fluid and other body fluids (14). Yang et al (15) demonstrated that serum exosome miR-135a and miR-384 were upregulated in patients with AD. The expression levels of miR-1291 and miR-597-5p were also increased in the cerebrospinal fluid of 
patients with AD (16). Recent studies have shown that some miRNAs in body fluids are overexpressed or expressed at low levels in numerous neurodegenerative diseases, such as AD, suggesting that miRNAs in body fluids could be used as novel biomarkers for the early diagnosis of these neurodegenerative diseases. The expression levels of miR-29a and miR-29c were significantly decreased in the serum of patients with Parkinson's disease (PD) and were negatively associated with the severity of PD (17). Dobrowolny et al (18) reported that serum miR-423-3p and miR-151a-5p were significantly downregulated in mild and terminal stages of the amyotrophic lateral sclerosis. Zhang et al (19) found that the expression level of serum miR-128 was increased in patients with AD, and this may serve as a promising diagnostic biomarker of AD. MiR-34c played important roles in the decline of synaptic function and memory impairment via the SYT1/ROS-JNK-p53 pathway (20). Hou et al (21) reported that miR-124 promoted tau protein phosphorylation, inducing the occurrence of $\mathrm{AD}$. It has also been confirmed that miRNA-22 could inhibit the release of inflammatory cytokines and improve the cognitive ability in a mouse model of AD (22). Increased mRNA expression levels of miR-326 in a mouse model of AD reduced the deposition of $A \beta$, and the contents of $A \beta 1-40$ and $A \beta 1-42$ (23).

Results of a previous study demonstrated that miR-4722-5p and miR-615-3p were AD-related biological markers from the analysis of AD samples at different stages of the disease (24). However, to the best of our knowledge, the mRNA expression levels and functional roles of miR-4722-5p and miR-615-3p in AD have not been reported. In the present study, the mRNA expression levels of miR-4722-5p and miR-615-3p in the serum of patients with AD were analyzed, and an A $\beta 25-35$-induced PC12 cell model was established to investigate their value as potential biomarkers for AD. This could provide a new theoretical basis and research direction for the early diagnosis, and further treatment of AD.

\section{Materials and methods}

Demographic data and clinical characteristics. A total of 33 patients with AD were recruited from the Department of Neurology, the Affiliated Hospital of Jiangsu University (Jiangsu, China), between November 2019 and June 2021. The patients with AD were diagnosed according to the 1984 National Institute of Neurological and Communicative Disorders and Stroke, and the AD and Related Disorders Association criteria (25). A total of 33 healthy volunteers, who were matched for age and sex, were admitted to the Health Examination Center of the Affiliated Hospital of Jiangsu University (Jiangsu, China) at the same period as previously described for patients with AD. Patient characteristics, including sex, age, years spent in education, and dementia risk factors, such as hypertension, diabetes, hyperhomocysteinemia, total cholesterol, low-density lipoprotein cholesterol, vitamin B12 and the folic acid of patients were determined using medical history and blood tests. Power analysis and sample size software (v15; NCSS, LLC) was used for sample size estimation to ensure the significance and reliability of the results. The mini-mental state examination (MMSE) scale is the most widely used cognitive function screening scale in clinical practice, and it can be used to assess the degree of cognitive impairment in patients with AD (26). The severity of AD, according to the MMSE scores, was defined as follows: Mild dementia (21 $\leq$ MMSE scores $\leq 26)$, moderate dementia (15 $\leq$ MMSE scores $\leq 20$ ) and severe dementia (MMSE scores <15) (27). The MMSE scores have been associated with literacy levels and are divided according to literacy levels (illiterate, $\leq 17$; primary school, $\leq 20$; junior high school or above, $\leq 24)$ (28). The clinical characteristics and demographic data of the patients with AD and the volunteers are shown in Table I. The present study was approved by the Scientific Research Ethics Committee of the Affiliated Hospital of Jiangsu University (Jiangsu, China), and written informed consent was obtained from each participant.

Serum sample collection. Venous blood samples were collected from all subjects after fasting for at least $8 \mathrm{~h}$ and immediately placed at $4^{\circ} \mathrm{C}$ for $1 \mathrm{~h}$. The upper serum was separated by centrifugation for $15 \mathrm{~min}$ at $1,500 \mathrm{x} \mathrm{g}$ and $4^{\circ} \mathrm{C}$, and stored at $-80^{\circ} \mathrm{C}$ until further analysis.

Cell culture and treatment. The rat pheochromocytoma cell line, PC12 was purchased from the Shanghai Institute of Cell Biology, Chinese Academy of Sciences. The cell line was cultured at $37^{\circ} \mathrm{C}$ in a humidified incubator containing $5 \%$ $\mathrm{CO}_{2}$, and maintained in high-glucose DMEM (Gibco; Thermo Fisher Scientific, Inc.), supplemented with 10\% FBS (Gibco; Thermo Fisher Scientific, Inc.) and $1 \%$ penicillin-streptomycin (Invitrogen; Thermo Fisher Scientific, Inc.). When the PC12 cells reached $90 \%$ confluence, the cells were washed with PBS (HyClone; Cytiva) and then collected with Trypsin-EDTA Solution (Biosharp, China). The cells were subsequently seeded in a 6 -well plate at a density of $5 \times 10^{5}$ cells each well, and treated with A $325-35$ (30 $\mu \mathrm{mol} / \mathrm{l} ; 24 \mathrm{~h}$; Sigma-Aldrich; Merck $\mathrm{KGaA}$ ) to induce cell damage. A $\beta 25-35$ was dissolved in PBS (HyClone; Cytiva) and maintained at $37^{\circ} \mathrm{C}$ for 7 days to allow for fibril formation before use.

$R N A$ extraction and reverse transcription-quantitative $P C R$ $(R T-q P C R)$. Serum RNA was extracted using RNApure Circulating Reagent (CoWin Biosciences), whilecellularRNA was isolated using a RNA-Quick Purification kit (ESscience Biotech), according to the manufacturer's instructions. The purity and concentration of the RNA was measured using a Titertek Berthold Micro Spectrophotometer (Berthold Technologies $\mathrm{GmbH}$ and $\mathrm{Co}$. KG), and the absorbance ratio of A260/A280 between 1.8 and 2.2 represented high purity. The cDNA synthesis reaction was conducted using the miRNA 1st Strand cDNA Synthesis kit (by Stem-Loop) (Vazyme Biotech Co., Ltd) and the reaction conditions of reverse transcription were $25^{\circ} \mathrm{C}$ for $5 \mathrm{~min}, 50^{\circ} \mathrm{C}$ for $15 \mathrm{~min}$ and $85^{\circ} \mathrm{C}$ for $5 \mathrm{~min}$. The ChamQ SYBR qPCR Master Mix kit (Vazyme Biotech Co., Ltd) and a QuantStudio5 Real-Time PCR System (Thermo Fisher Scientific, Inc.) were used to amplify the cDNA, which was conducted in a 96-well plate. Each sample was performed with 3 duplicate wells and U6 served as the internal reference control. The following thermocycling conditions were used: Initial denaturation at $95^{\circ} \mathrm{C}$ for $30 \mathrm{sec}$; followed by 40 cycles at $95^{\circ} \mathrm{C}$ for $10 \mathrm{sec}, 56^{\circ} \mathrm{C}$ for $30 \mathrm{sec}$ and $72^{\circ} \mathrm{C}$ for $60 \mathrm{sec}$; and final extension at $95^{\circ} \mathrm{C}$ for $15 \mathrm{sec}, 60^{\circ} \mathrm{C}$ for $60 \mathrm{sec}$ and $95^{\circ} \mathrm{C}$ for 
Table I. Comparisons of clinical characteristics and demographic data between patients with AD and the healthy controls.

\begin{tabular}{|c|c|c|c|c|c|c|}
\hline Characteristic & $\begin{array}{l}\text { Healthy controls } \\
\qquad(\mathrm{n}=33)\end{array}$ & $\begin{array}{l}\text { Patients with AD } \\
\quad(\mathrm{n}=33)\end{array}$ & $\mathrm{F}$ & $\mathrm{X}^{2}$ & $\mathrm{Z}$ & P-value \\
\hline Mean age $\pm \mathrm{SD}$, years & $42 \pm 6.85$ & $73.58 \pm 8.41$ & 0.487 & $\mathrm{~N} / \mathrm{A}$ & N/A & 0.331 \\
\hline Female, n (\%) & $(57.58)$ & $16(48.48)$ & $\mathrm{N} / \mathrm{A}$ & 0.547 & N/A & 0.459 \\
\hline Male, n (\%) & $(42.42)$ & $17(51.52)$ & & & & \\
\hline \multicolumn{7}{|l|}{ Education, n (\%) } \\
\hline High school and above & $(15.15)$ & $1(3.03)$ & 2.889 & $\mathrm{~N} / \mathrm{A}$ & N/A & 0.089 \\
\hline Junior high school and above & $(84.85)$ & $32(96.97)$ & & & & \\
\hline Hypertension, n (\%) & $(54.55)$ & $19(57.58)$ & N/A & 0.062 & N/A & 0.804 \\
\hline Diabetes, $\mathrm{n}(\%)$ & $(39.40)$ & $15(45.45)$ & N/A & 0.248 & N/A & 0.618 \\
\hline HHcy, n (\%) & $(48.48)$ & $11(33.33)$ & $\mathrm{N} / \mathrm{A}$ & 0.262 & N/A & 0.211 \\
\hline Mean \pm SD total cholesterol, $\mathrm{mmol} / \mathrm{l}$ & $86 \pm 0.95$ & $4.52 \pm 0.98$ & 0.048 & $\mathrm{~N} / \mathrm{A}$ & N/A & 0.169 \\
\hline Mean \pm SD LDL-C, mmol/l & $88 \pm 0.70$ & $2.62 \pm 0.82$ & 0.856 & $\mathrm{~N} / \mathrm{A}$ & N/A & 0.178 \\
\hline Mean $\pm \mathrm{SD}$ vitamin $\mathrm{B} 12, \mathrm{pg} / \mathrm{ml}$ & $36 \pm 206.93$ & $401.33 \pm 161.71$ & 0.878 & $\mathrm{~N} / \mathrm{A}$ & $\mathrm{N} / \mathrm{A}$ & 0.712 \\
\hline Mean \pm SD folic acid, $\mathrm{ng} / \mathrm{ml}$ & $6.09 \pm 2.65$ & $5.09 \pm 1.91$ & 2.667 & $\mathrm{~N} / \mathrm{A}$ & N/A & 0.083 \\
\hline Mean (IQR) MMSE scores & $28(27-30)$ & $16(12-17)$ & $\mathrm{N} / \mathrm{A}$ & $\mathrm{N} / \mathrm{A}$ & -7.017 & $<0.001$ \\
\hline
\end{tabular}

AD, Alzheimer's disease; HHcy, hyperhomocysteine; LDL-C, low-density lipoprotein cholesterol; MMSE, mini-mental state examination; IQR, interquartile range; N/A, not applicable.

$15 \mathrm{sec}$. The relative mRNA expression levels of the miRNA were calculated using the $2^{-\Delta \Delta C q}$ method (29). All the primers were designed and synthesized by Sangon Biotech Co., Ltd., using stem-loop methods. Universal reverse primers (URP) were used as the reverse primers for miR-4722-5p and miR-615-3p. The following specific sequences were used for RNA extracted from cells and serum: miR-4722-5p forward, 5'-GGCAGGAGGGCTGTGCC-3'; URP, 5'-AGTGCAGGG TCCGAGGTATT-3'; U6 forward, 5'-AGAGAAGATTAG CATGGCCCCTG-3', and reverse, 5'-ATCCAGTGCAGG GTCCGAGG-3'. The forward sequences, 5'-CGTCCGAGC CTGGGTCTC-3' and 5'-GGGGGTCCCCGGTGCT-3' were used for serum and cell samples, respectively.

miRNA target gene prediction. miRWalk version 3.0 (http://mirwalk.umm.uni-heidelberg.de/) is a comprehensive database to predict the target genes of miRNAs, and contains information of miRNA target genes from humans, mice, rats and several other species. For example, the sequences of miR-211 from different species, including humans, mice, rats, fish, dogs and cows can be found in the miRWalk database. The target genes of miR-4722-5p and miR-615-3p were predicted using the miRWalk database. The potential target genes were screened under the following conditions: miRNA binding region 3'-untranslated region scores $\geq 1$.

Gene Ontology (GO) and Kyoto Encyclopedia of Genes and Genomes (KEGG) enrichment analyses of the miRNA target genes. GO and KEGG enrichment analyses of the miRNA target genes were performed using The Database for Annotation, Visualization and Integrated Discovery Bioinformatics Resources database version 6.8 (https://david. ncifcrf.gov/). The R language analysis package was used to display the top $10 \mathrm{GO}$ and the top $15 \mathrm{KEGG}$ enrichment pathway information. The significant difference of enrichment analysis was set as $\mathrm{P}<0.05$.

Statistical analysis. SPSS version 22.0 software (IBM Corp.) and GraphPad Prism version 8.0.2 software (GraphPad Software, Inc.) were used for statistical analysis. Numerical data are presented as a number or percentage, and either a $\chi^{2}$ test or Fisher's exact test was used for comparison between patients with $\mathrm{AD}$ and the healthy controls. Continuous data are expressed as the mean $\pm \mathrm{SD}$ or the median (interquartile range), and the difference between patients with $\mathrm{AD}$ and healthy controls was analyzed using either a t-test or the Mann-Whitney U test. The Spearman correlation coefficient was used to analyze the correlation between the expression of the two miRNAs and MMSE scores. A receiver operating characteristic curve (ROC) was used to evaluate the specificity and sensitivity of miR-4722-5p and miR-615-3p in AD. A binary logistic regression model was used to predict the diagnostic value for $\mathrm{AD}$ by combining the two miRNAs. $\mathrm{P}<0.05$ was considered to indicate a statistically significant difference.

\section{Results}

Clinical characteristics and demographic data. A total of 33 patients with $\mathrm{AD}$ (17 males and 16 females) and 33 healthy individuals (14 males and 19 females) were recruited into the present study. The average age of the patients with $\mathrm{AD}$ was $73.58 \pm 8.41$ years, while that in the healthy individuals was $75.42 \pm 6.85$ years. The two groups exhibited no significant differences in sex, age or years spent in education, and exhibited no significant differences in dementia risk factors, including hypertension, diabetes, hyperhomocysteinemia, total cholesterol, low-density lipoprotein cholesterol, vitamin B12 or folic acid $(\mathrm{P}>0.05)$, but there were statistically significant 
differences between the two groups in MMSE scores $(\mathrm{P}<0.001)$ (Table I).

Relative expression level of miR-4722-5p and miR-615-3p in patients with $A D$. The melting curve of RT-qPCR was unimodal, which ensured the specificity of the amplified products. The results showed that the mRNA expression levels of miR-4722-5p (Z,-3.918) and miR-615-3p (Z, -4.675) in the patients with $\mathrm{AD}$ were both higher compared with that in the healthy controls $(\mathrm{P}<0.001)$ (Fig. 1A and B). The mRNA expression levels of miR-4722-5p ( $t$, 6.169) and miR-615-3p (t, 8.345) in the $\mathrm{A} \beta 25-35$-treated $\mathrm{PC} 12$ cells were also higher compared with that in the control group $(\mathrm{P}<0.05)$ (Fig. $1 \mathrm{C}$ and $\mathrm{D})$.

Correlations between the relative $m R N A$ expression levels of miR-4722-5p and miR-615-3p, and MMSE scores. The relative mRNA expression levels of serum miR-4722-5p and miR-615-3p were negatively correlated with MMSE scores according to the analysis of Spearman's correlation coefficient. The relative expression levels of serum miR-4722-5p (r, -0.771; $\mathrm{P}<0.0001$; Fig. 2A) and miR-615-3p (r, $-0.780 ; \mathrm{P}<0.0001$; Fig. $2 \mathrm{~B}$ ) were higher in patients with $\mathrm{AD}$ and lower MMSE scores.

Diagnostic power of miR-4722-5p and miR-615-3p in patients with $A D$. The ROC curve is mainly used to evaluate the diagnostic value of a certain index to obtain the best index threshold (30). The area under the curve (AUC) of serum miR-4722-5p was 0.781 , the sensitivity was 0.697 and the specificity was 0.848 (Fig. 3A). The AUC of serum miR-615-3p was 0.835 , the sensitivity was 0.727 and the specificity was 0.909 (Fig. 3B). The logistic regression model showed that the AUC of both miRNAs combined was 0.870 , the sensitivity was 0.697 and the specificity was 0.939 (Fig. 3C). The sensitivity and specificity of miR-615-3p for the diagnosis of AD were higher compared with that in miR-4722-5p for the diagnosis of $\mathrm{AD}$. In addition, the specificity of the two miRNAs combined for $\mathrm{AD}$ was higher compared with that for each miRNA alone. The results of the ROC curve analysis are shown in Table $\Pi$.

GO enrichment analysis of the miRNA target genes. GO is a database established by the Association of Gene Ontology Consortium and includes three main categories: Biological process (BP), cellular component (CC) and molecular function (MF) (31). BP analysis of the target genes of miR-4722-5p showed that these genes were mainly involved in 'cell division', 'protein folding', 'sister chromatid cohesion', 'actin cytoskeleton organization' and 'cAMP catabolic process' (Fig. 4A). $\mathrm{CC}$ analysis showed that the target genes of miR-4722-5p were mainly located in the 'cytoplasm', 'cytosol', 'nucleoplasm' and 'membrane' (Fig. 4B). MF analysis indicated that the target genes of miR-4722-5p performed the functions of 'protein binding', 'metal ion binding', 'nucleic acid binding' and 'GTPase activator activity' (Fig. 4C). BP analysis of the target genes of miR-615-3p indicated that these genes mainly participated in the 'regulation of transcription, DNA-templated', 'protein phosphorylation', 'protein sumoylation' and 'protein complex assembly' (Fig. 4D). The target genes of miR-615-3p were found in the 'nucleus', 'cytoplasm', 'cytosol' and 'nucleoplasm' (Fig. 4E) and played roles in 'protein binding', 'metal
A
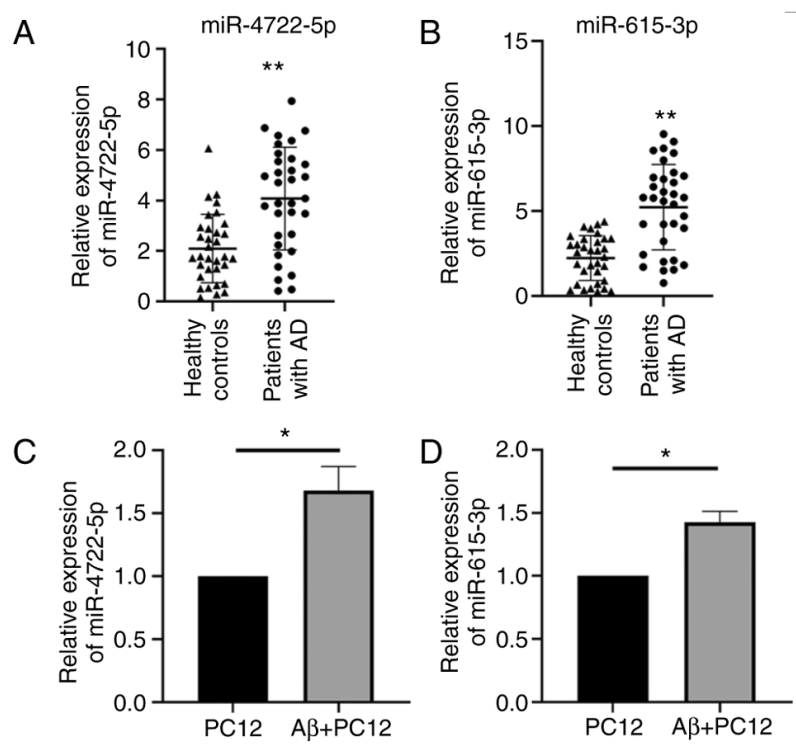

Figure 1. mRNA expression level of miR-4722-5p and miR-615-3p in patients with $\mathrm{AD}$, and $\mathrm{A} \beta 25-35$-treated PC12 cells. The mRNA expression levels of serum (A) miR-4722-5p (Mann-Whitney U test) and (B) miR-615-3p (Mann-Whitney U test) were higher in patients with AD. The mRNA expression levels of (C) miR-4722-5p (single-sample t-test) and (D) miR-615-3p (single-sample t-test) in the A $\beta 25-35$-treated PC12 cells were increased compared with that in the control group. ${ }^{*} \mathrm{P}<0.05$. ${ }^{* *} \mathrm{P}<0.001$. miR, microRNA; $\mathrm{A} \beta, \beta$-amyloid; AD, Alzheimer's disease.

ion binding', 'zinc ion binding' and 'transcription factor activity, sequence-specific DNA binding' (Fig. 4F).

KEGG enrichment analysis of miRNA target genes. KEGG is a database integrating genomic, chemical and system functional information (32). KEGG enrichment analysis of the target genes for miR-4722-5p showed that these genes were mainly involved in 'proteoglycans in cancer', 'purine metabolism', 'neurotrophin signaling pathway' and 'mTOR signaling pathway' (Fig. 5A). The target genes for miR-615-3p were associated with 'endocytosis', 'insulin resistance', 'Ras signaling pathway' and 'FoxO signaling pathway' (Fig. 5B).

\section{Discussion}

$\mathrm{AD}$ is a chronic progressive neurodegenerative disease that affects $\sim 47$ million people worldwide, and that number is expected to increase by $62 \%$ by 2030 (33). It has been hypothesized that the $A \beta$ waterfall theory (34), the tau protein theory (35), oxidative stress (36), inflammatory mechanisms (37), mitochondrial dysfunction (38) and other theories are involved in the pathogenesis of AD. An increase in $A \beta$ levels in the brain may lead to $A \beta$ aggregation into oligomers, thus initiating a series of events leading to cell dysfunction and death (39). Abnormal phosphorylation, aggregation and proteolysis of the tau protein in a pre-tangle stage of neurofibrillary degeneration has also been proved to be an early and crucial event in the pathogenesis of AD (40). It has been found that reduction or loss of trigger receptor expressed on myeloid cells 2 function in a mouse model of tauopathy was neuroprotective, reducing gliosis and neuroinflammation (41). The clinical diagnosis of AD mainly depends on the clinical manifestations 
Table II. Results of receiver operating characteristic curve analysis.

\begin{tabular}{lcccrr}
\hline miRNA & AUC $(95 \% \mathrm{CI})$ & Sensitivity & Specificity & Cut-off value & P-value \\
\hline miR-4722-5p & $0.781(0.666-0.895)$ & 0.697 & 0.848 & 3.455 & $<0.001$ \\
miR-615-3p & $0.835(0.734-0.935)$ & 0.727 & 0.909 & 3.976 & $<0.001$ \\
miR-4722-5p and miR-615-3p & $0.870(0.780-0.959)$ & 0.697 & 0.939 & 0.615 & $<0.001$ \\
\hline
\end{tabular}

AUC, area under the curve; CI, confidence interval; miRNA/miR, microRNA.
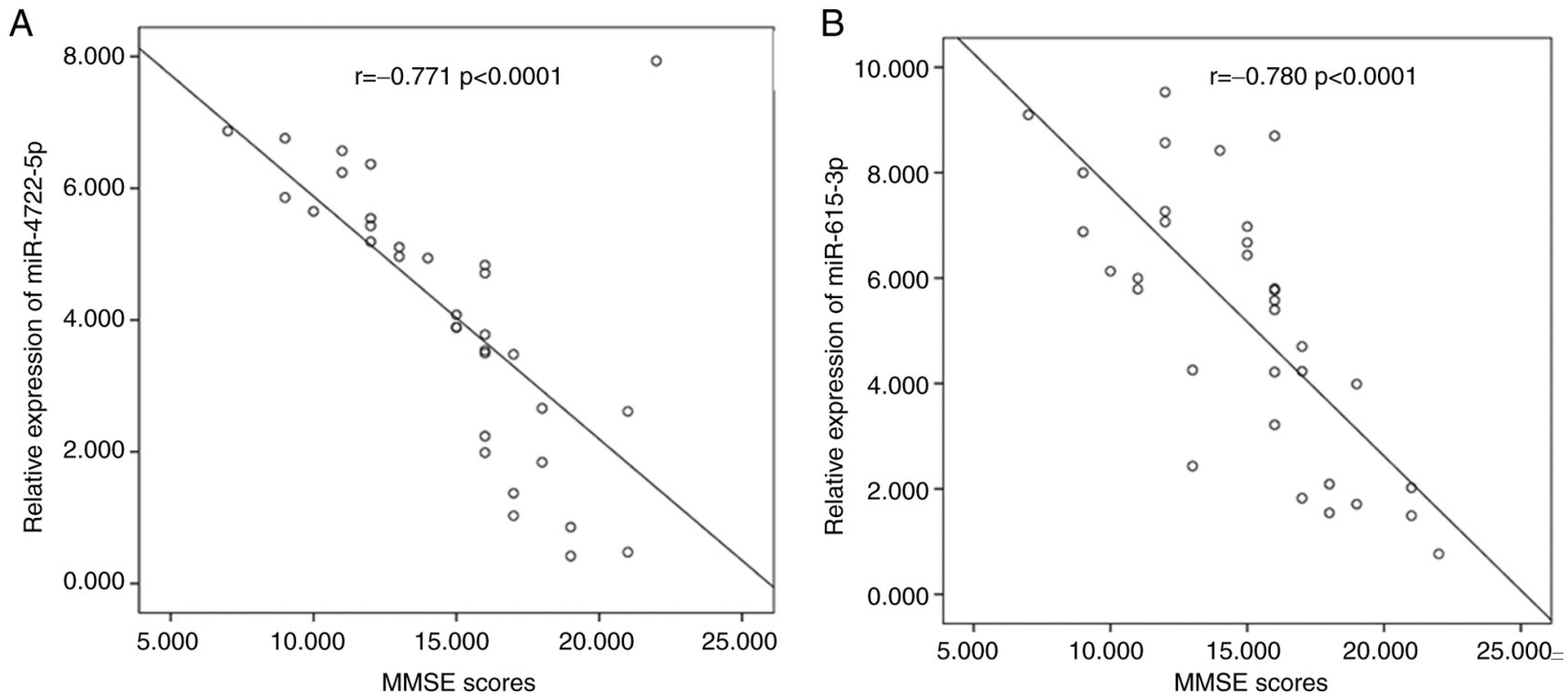

Figure 2. Correlation between miRNA expression levels and MMSE scores in patients with AD. Correlation between mRNA expression levels of (A) miR-4722-5p and (B) miR-615-3p, and MMSE scores in patients with AD. AD, Alzheimer's disease; miR, microRNA; MMSE, mini-mental state examination.
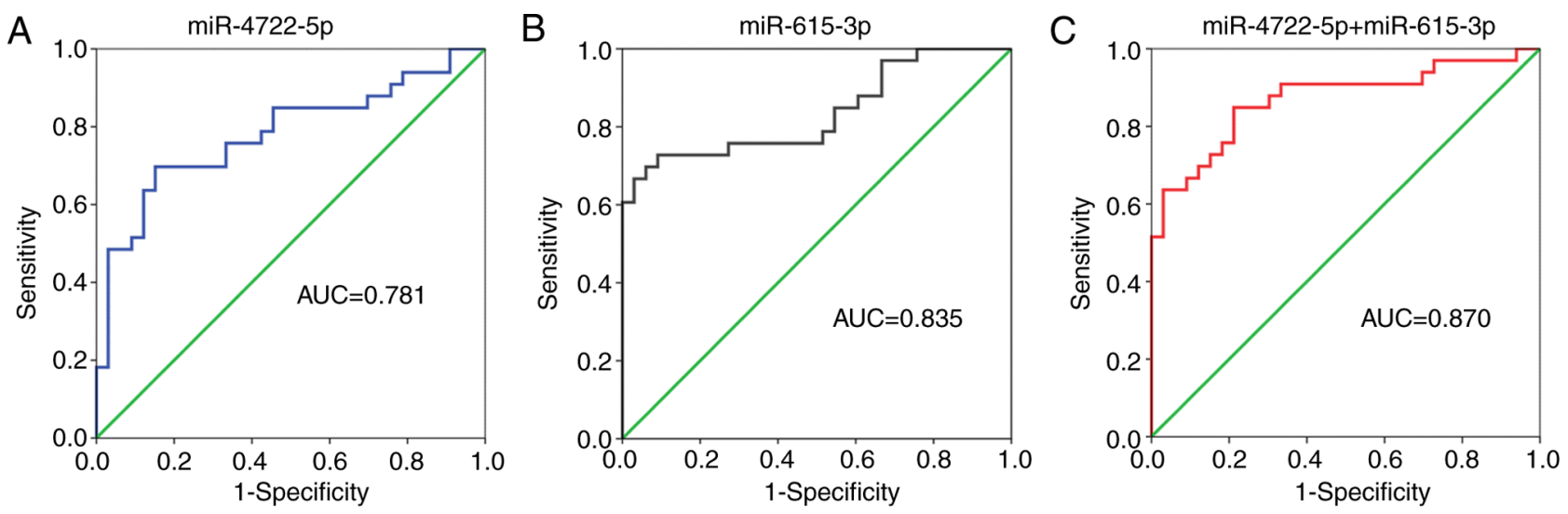

Figure 3. ROC curve analysis of serum miRNAs. ROC curve analysis of serum (A) miR-4722-5p and (B) miR-615-3p, and (C) miR-4722-5p and miR-615-3p. miR, microRNA; ROC, receiver operating characteristic.

of the patient, neuropsychological scales, genetic testing and imaging techniques (42). Studies have shown that $A \beta$-positron emission tomography (PET) has a certain value in the diagnosis of AD (43). However, A $\beta$-PET has not been widely used in the diagnosis of AD in a clinical setting as the method for analyzing the fluorodeoxyglucose-PET data has not reached the same degree of standardization and the cost is high. It has also been confirmed that $\mathrm{A} \beta 42$, the ratio of $\mathrm{A} \beta 42 / \mathrm{A} \beta 40$, total tau protein and phosphorylated tau protein in the cerebrospinal fluid are important biomarkers for the diagnosis of AD (44). However, lumbar puncture is an invasive examination (45) and it is difficult to obtain the cooperation of the patient or the family members. miRNAs are non-invasive biomarkers and their specific expressions have been associated with the 


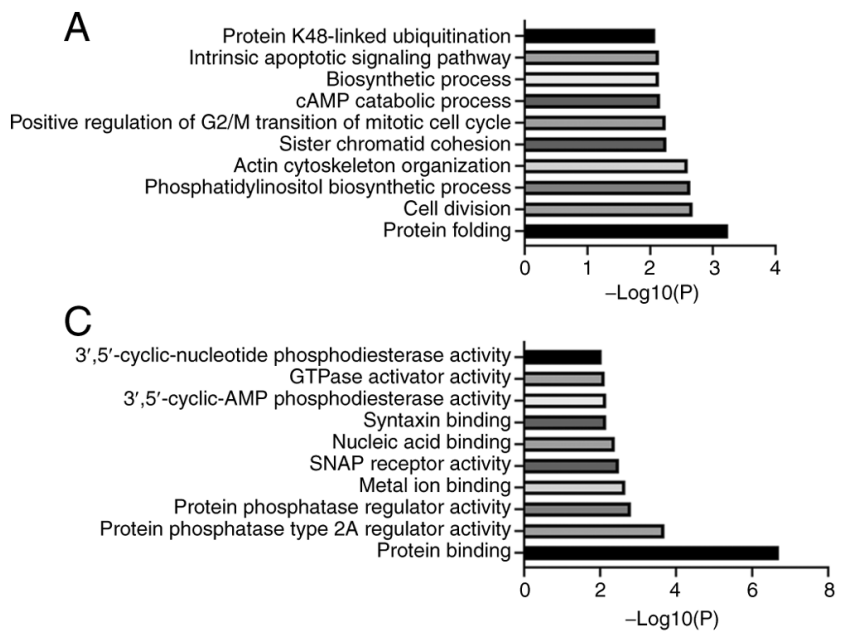

E

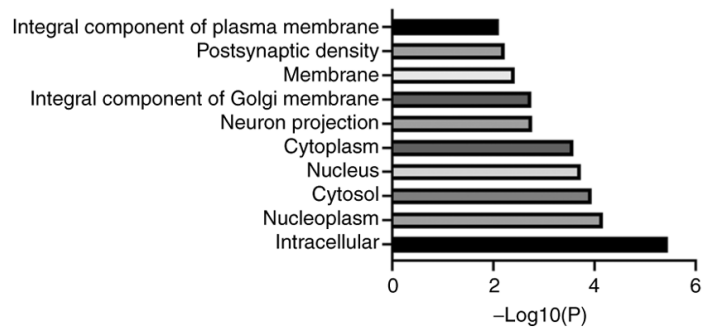

B

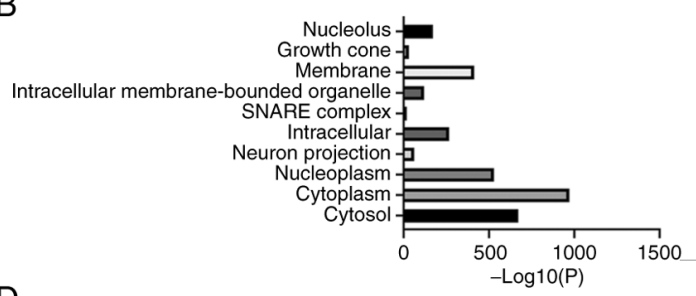

D

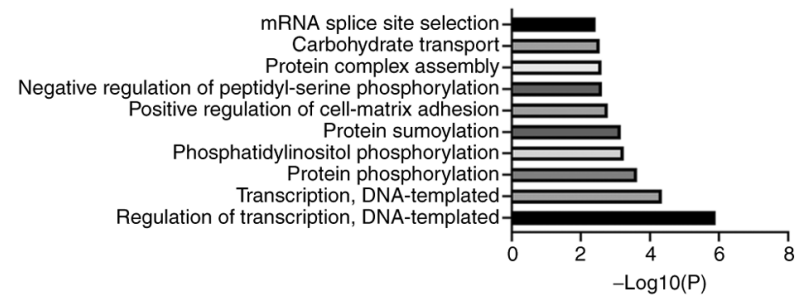

$\mathrm{F}$

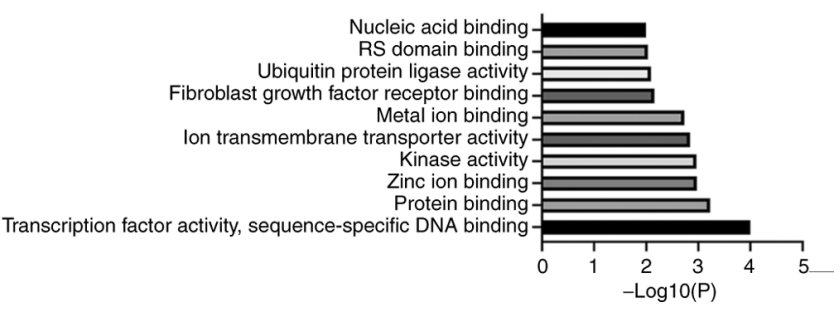

Figure 4. Gene Ontology enrichment analysis of the target genes of both miRNAs. (A) Biological processes, (B) cellular component and (C) molecular function of the target genes for miR-4722-5p. (D) Biological processes, (E) cellular component and (F) molecular function of the target genes for miR-615-3p. miR, microRNA.

pathogenesis of $\mathrm{AD}(46)$. However, finding miRNAs expressed only in the serum of patients with AD is not easy.

In the present study, the mRNA expression levels of miR-4722-5p and miR-615-3p in the serum of patients with $\mathrm{AD}$, and the $\mathrm{A} \beta 25-35$-induced $\mathrm{PC} 12$ cell model were higher compared with that in the control groups. GO analysis indicated that the target genes of miR-4722-5p might be involved in the cAMP catabolic process. cAMP produced by ATP from the adenylate cyclase family is the second messenger required for long-term enhancement and memory consolidation (47,48). Nassireslami et al (49) demonstrated that the upregulation of cAMP analogues activated the cAMP/PKA signaling pathway, and improved synaptic plasticity and memory deficits. In addition, the cAMP/PKA signaling pathway can reduce the production of tau protein and improve cognitive deficits (50). KEGG analysis of the target genes for miR-4722-5p revealed that they were associated with the mTOR, neurotrophin, prolactin, 'SNARE interactions in vesicular transport', 'proteoglycans in cancer', 'non-alcoholic fatty liver disease', 'purine metabolism', 'pancreatic cancer', 'morphine addiction', phosphatidylinositol, sphingolipid, 'one carbon pool by folate', 'leukocyte transendothelial migration', 'choline metabolism in cancer' and 'dopaminergic synapse'. mTOR is the main regulator of autophagy and plays an important role in neurodegenerative diseases $(51,52)$. mTOR inhibitors, including rapamycin, have been shown to be effective in improving cognitive deficits in AD (53). Therefore, it was hypothesized that miR-4722-5p might regulate the mTOR pathway, inducing the occurrence of $\mathrm{AD}$.
It has been confirmed that miR-615-3p was associated with the occurrence and development of numerous diseases, such as esophageal, gastric and non-small lung cancers. The expression of miR-615-3p was upregulated in the brain of patients with Huntington's disease, and was associated with the pathogenesis of HD (54). Miyamoto et al (55) found that the enhancement of miR-615-3p expression levels might be of therapeutic benefit for nonalcoholic fatty liver disease by inhibiting palmitate-induced hepatocyte lipoapoptosis. Feng et al (56) demonstrated that miR-615-3p could inhibit the apoptosis of epileptiform hippocampal neurons via the PI3K/Akt/mTOR pathway. GO analysis of the target genes for miR-615-3p was associated with BP, such as transcription, 'protein phosphorylation', 'protein complex assembly', 'phosphatidylinositol phosphorylation', 'protein sumoylation', 'positive regulation of cell-matrix adhesion', 'negative regulation of peptidyl-serine phosphorylation', 'carbohydrate transport', 'mRNA splice site selection' and other biological processes. Wang et al (57) reported that miR-615-3p promoted gastric cancer proliferation and migration by suppressing the expression of CUGBP- and ETR-3-like family 2. KEGG analysis demonstrated that its target genes were engaged in regulating FoxO, ErbB, melanoma, 'non-small cell lung cancer', glioma, 'ubiquitin mediated proteolysis', 'axon guidance', 'protein processing in endoplasmic reticulum', 'thyroid hormone', endocytosis, 'transcriptional misregulation in cancer', 'insulin resistance' and Ras signaling pathway. FoxO transcription factors have been associated with nerve cell survival, and neuronal signal transmission exists in the hippocampus, amygdala and nucleus accumbens $(58,59)$. FoxO transcription factors have also been 
A

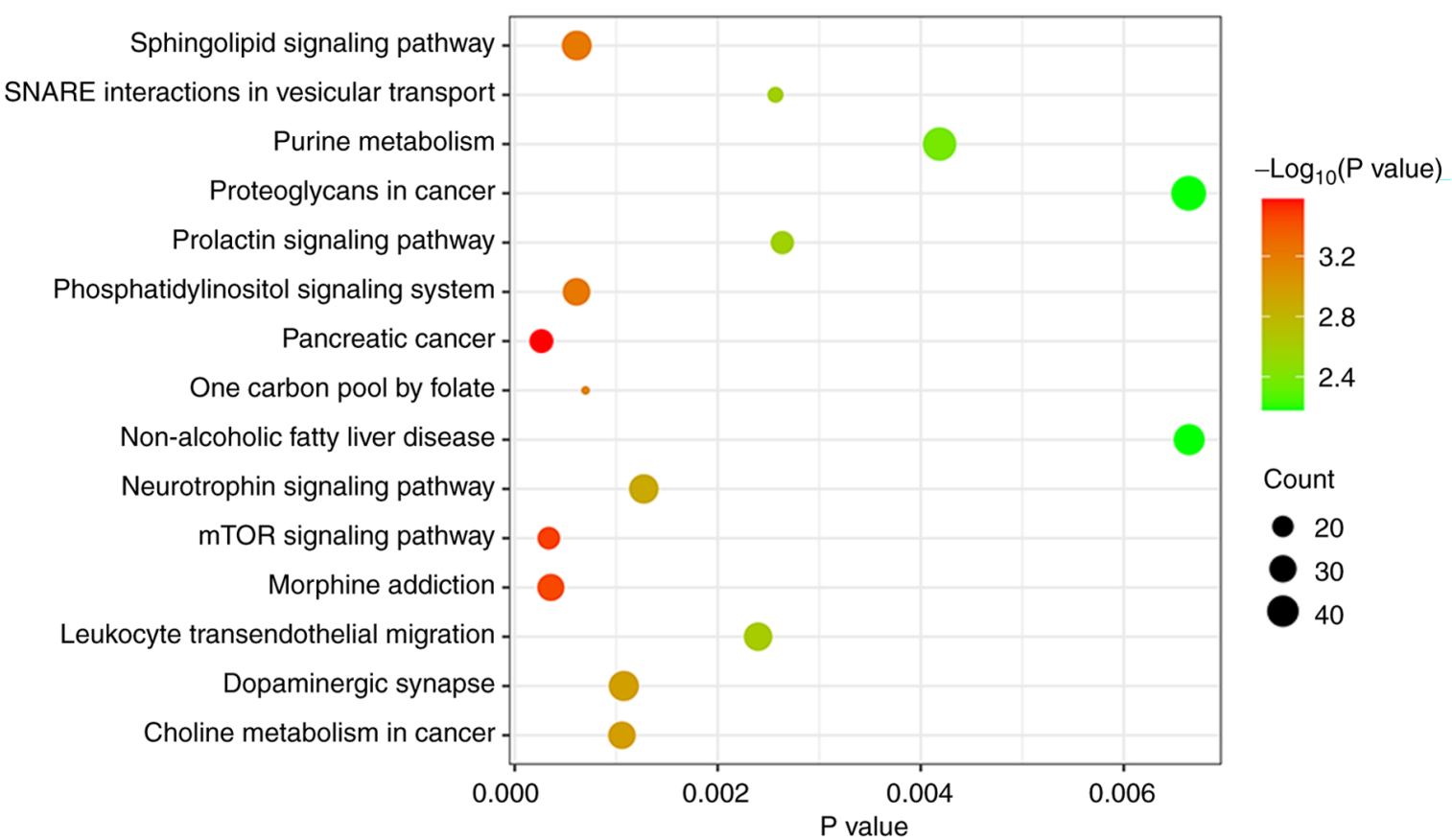

B

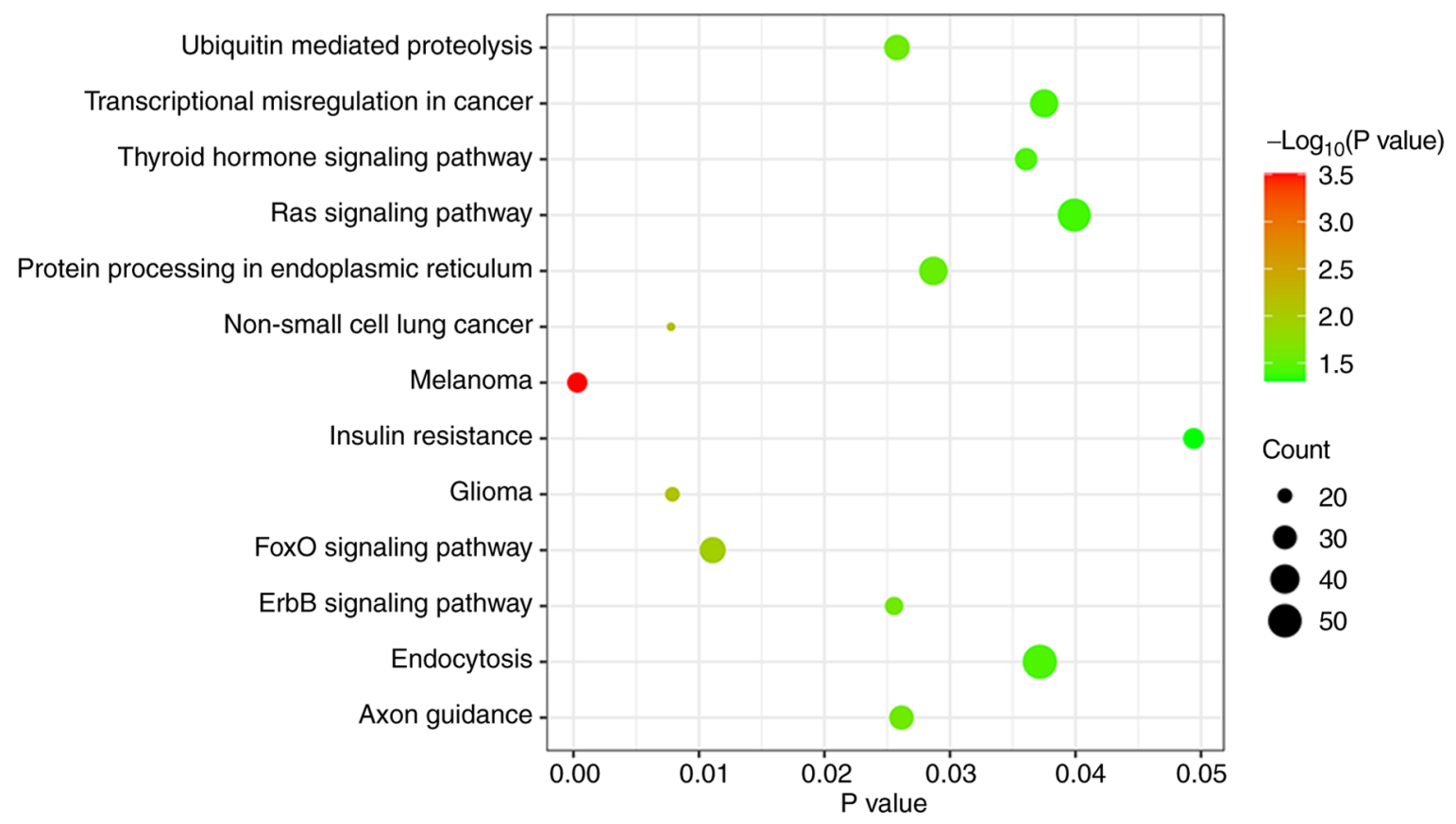

Figure 5. KEGG enrichment analysis of miRNA target genes. KEGG enrichment analysis of the target genes of (A) miR-4722-5p and (B) miR-615-3p. miR, microRNA; KEGG, Kyoto Encyclopedia of Genes and Genomes.

identified as potential targets for a variety of neurodegenerative diseases, such as AD, Parkinson's disease and Huntington's disease (60). Maiese (61) demonstrated that FoxO transcription factors could not only promote apoptotic cell death in the nervous system but also offer protection against degenerative disease through the induction of autophagy that could lead to dementia. In some circumstances, $A \beta$ could induce the dephosphorylation and mitochondrial translocation of FoxO3a leading to mitochondrial dysfunction (62). Increased activity of FoxO could result in the apoptosis and autophagy in $A \beta$-induced neuron death (63). EGFR, a transmembrane glycoprotein, is a member of the ErbB receptor tyrosine kinase superfamily (64). Stupack et al (65) found that SORLA, a transmembrane transporter associated with the risk of $\mathrm{AD}$, promoted neurite regeneration by activating EGF receptors. Mansour et al (66) proposed that EGFR inhibitors had neuroprotective effects on AD models. Therefore, miR-615-3p may be crucial to the pathogenesis of $\mathrm{AD}$ by regulating the FoxO and ErbB signaling pathways.

The present study is preliminary and some limitations must be acknowledged. Firstly, the basic information of the individuals recruited into the study were matched as best as 
possible, including sex and age to minimize the influence of confounding factors. Nevertheless, the results may be limited due to the small sample size, different lifestyles of the patients, genetic variation and external effects, such as regional differences among patients with AD. Replication of the results in further studies with larger sample sizes is required. Secondly, Parkinson's disease is another well-known neurodegenerative disease. Therefore, it is necessary to detect the expression levels of miR-4722-5p and miR-615-3p in patients with Parkinson's disease in future studies. Moreover, results of the present study demonstrated that miR-4722-5p and miR-615-3p are highly expressed in the AD cell model and the serum of patients with ADs. Thus, animal models will be used in future investigations to verify the conclusions of the present study, and to investigate the specific mechanisms underlying miR-4722-5p and miR-615-3p in AD. In addition, previous studies have demonstrated that the sequences of most miRNAs are highly conserved among different species $(67,68)$. To date, the rat sequence of miR-4722-5p has not been published in the database; therefore, human primers were used for RT-qPCR from RNA extracted from the PC12 cell line. Finally, the mRNA expression levels of miR-495-3p in the serum of patients with AD and healthy controls showed no statistically significant difference in our previous experiment (data not published). However, there was no relevant reports on the mRNA expression levels of miR-495-3p in patients with AD in current studies. Eun et al (69) demonstrated that the overexpression of miR-495-3p could cause the death of gastric cancer cells. Further investigation into other miRNAs in the serum of patients with $\mathrm{AD}$ and healthy controls will be a new goal for future research.

In summary, it was first confirmed that the mRNA expression levels of miR-4722-5p and miR-615-3p were increased in patients with $\mathrm{AD}$, and the $\mathrm{A} \beta 25-35$-induced PC12 cell model compared with that in the control groups in the present study. Furthermore, MMSE scores were negatively correlated with the mRNA expression levels of the two miRNAs. These findings suggest that miR-4722-5p and miR-615-3p may be potential biomarkers for the diagnosis of $\mathrm{AD}$; however, the specific mechanism requires further investigation.

\section{Acknowledgements}

The authors would like to thank Professor Ming Yu (Chief Physician at the Department of Neurology, the Affiliated Hospital of Jiangsu University) and Yuhao $\mathrm{Xu}$ (Resident Physician at the Department of Neurology, the Affiliated Hospital of Jiangsu University) for their guidance on the design of the experimental scheme and the revision of the paper.

\section{Funding}

This study was supported by the Zhenjiang Key Research and Development Plan (Social Development) (grant no. SH2019036).

\section{Availability of data and materials}

The datasets used and/or analyzed during the current study are available from the corresponding author on reasonable request.

\section{Authors' contributions}

MY and YL conceived and designed the study. YL and YX completed all the experiments and analyzed the experimental data. MY, YL and YX wrote and revised the manuscript. MY and YL confirmed the authenticity of all the raw data. All authors read and approved the final manuscript.

\section{Ethics approval and consent to participate}

The study was reviewed and approved by the Scientific Research Ethics Committee of the Affiliated Hospital of Jiangsu University (Jiangsu, China), and written informed consent was provided by each participant.

\section{Patient consent for publication}

Not applicable.

\section{Competing interests}

The authors declare that they have no competing interests.

\section{References}

1. Eratne D, Loi SM, Farrand S, Kelso W, Velakoulis D and Looi JC: Alzheimer's disease: Clinical update on epidemiology, pathophysiology and diagnosis. Australas Psychiatry 26: 347-357, 2018.

2. Di Resta C and Ferrari M: New molecular approaches to Alzheimer's disease. Clin Biochem 72: 81-86, 2019.

3. Takeda S: Progression of Alzheimer's disease, tau propagation, and its modifiable risk factors. Neurosci Res 141: 36-42, 2019.

4. Jiang L, Dong H, Cao H, Ji X, Luan S and Liu J: Exosomes in pathogenesis, diagnosis, and treatment of Alzheimer's Disease. Med Sci Monit 25: 3329-3335, 2019.

5. Tian X, Wang J, Dai J, Yang L, Zhang L, Shen S and Huang P: Hyperbaric oxygen and Ginkgo Biloba extract inhibit A $325-35$-induced toxicity and oxidative stress in vivo: A potential role in Alzheimer's disease. Int J Neurosci 122: 563-569, 2012.

6. Karch CM, Cruchaga C and Goate AM: Alzheimer's disease genetics: from the bench to the clinic. Neuron 83: 11-26, 2014.

7. Pereira JB, Westman E and Hansson O: Association between cerebrospinal fluid and plasma neurodegeneration biomarkers with brain atrophy in Alzheimer's disease. Neurobiol Aging 58: 14-29, 2017.

8. Pradhan R, Yadav SK, Prem NN, Bhagel V, Pathak M, Shekhar S, Gaikwad S, Dwivedi SN, Bal CS, Dey AB and Dey S: Serum FOXO3A: A ray of hope for early diagnosis of Alzheimer's disease. Mech Ageing Dev 190: 111290, 2020.

9. Goetzl EJ, Boxer A, Schwartz JB, Abner EL, Petersen RC, Miller BL and Kapogiannis D: Altered lysosomal proteins in neural-derived plasma exosomes in preclinical Alzheimer disease. Neurology 85: 40-47, 2015.

10. Abdullah M, Kimura N, Akatsu H, Hashizume Y, Ferdous T, Tachita T, Iida S, Zou K, Matsubara E and Michikawa M: Flotillin is a novel diagnostic blood marker of Alzheimer's Disease. J Alzheimers Dis 72: 1165-1176, 2019.

11. Briggs R, Kennelly SP and O'Neill D: Drug treatments in Alzheimer's disease. Clin Med (Lond) 16: 247-253, 2016.

12. Sancesario GM and Bernardini S: Alzheimer's disease in the omics era. Clin Biochem 59: 9-16, 2018.

13. Serpente M, Fenoglio C, D'Anca M, Arcaro M, Sorrentino F, Visconte C, Arighi A, Fumagalli GG, Porretti L, Cattaneo A, et al: MiRNA profiling in plasma neural-derived small extracellular vesicles from patients with Alzheimer's Disease. Cells 9: 1443, 2020.

14. Silvestro S, Bramanti P and Mazzon E: Role of miRNAs in Alzheimer's Disease and possible fields of application. Int J Mol Sci 20: 3979, 2019. 
15. Yang TT, Liu CG, Gao SC, Zhang Y and Wang PC: The serum exosome derived MicroRNA-135a, -193b, and -384 Were potential Alzheimer's Disease Biomarkers. Biomed Environ Sci 31 87-96, 2018.

16. Lusardi TA, Phillips JI, Wiedrick JT, Harrington CA, Lind B, Lapidus JA, Quinn JF and Saugstad JA: MicroRNAs in human cerebrospinal fluid as biomarkers for Alzheimer's Disease. J Alzheimers Dis 55: 1223-1233, 2017.

17. Bai X, Tang Y, Yu M, Wu L, Liu F, Ni J, Wang Z, Wang J, Fei J, Wang W, et al: Downregulation of blood serum microRNA 29 family in patients with Parkinson's disease. Sci Rep 7: 5411, 2017.

18. Dobrowolny G, Martone J, Lepore E, Casola I, Petrucci A, Inghilleri M, Morlando M, Colantoni A, Scicchitano BM, Calvo A, et al: A longitudinal study defined circulating microRNAs as reliable biomarkers for disease prognosis and progression in ALS human patients. Cell Death Discov 7: 4 2021.

19. Zhang M, Han W, Xu Y, Li D and Xue Q: Serum miR-128 Serves as a potential diagnostic biomarker for Alzheimer's Disease. Neuropsychiatr Dis Treat 17: 269-275, 2021.

20. Shi Z, Zhang K, Zhou H, Jiang L, Xie B, Wang R, Xia W, Yin Y, Gao Z, Cui D, et al: Increased miR-34c mediates synaptic deficits by targeting synaptotagmin 1 through ROS-JNK-p53 pathway in Alzheimer's Disease. Aging Cell 19: e13125, 2020.

21. Hou TY, Zhou Y, Zhu LS, Wang X, Pang P, Wang DQ, Liuyang ZY, Man H, Lu Y, Zhu LQ and Liu D: Correcting abnormalities in miR-124/PTPN1 signaling rescues tau pathology in Alzheimer's disease. J Neurochem 154: 441-457, 2020.

22. Han C, Guo L, Yang Y, Guan Q, Shen H, Sheng Y and Jiao Q: Mechanism of microRNA-22 in regulating neuroinflammation in Alzheimer's disease. Brain Behav 10: e01627, 2020.

23. He B, Chen W, Zeng J, Tong W and Zheng P: MicroRNA-326 decreases tau phosphorylation and neuron apoptosis through inhibition of the JNK signaling pathway by targeting VAV1 in Alzheimer's disease. J Cell Physiol 235: 480-493, 2020.

24. Soleimani Zakeri NS, Pashazadeh S and MotieGhader H: Gene biomarker discovery at different stages of Alzheimer using gene co-expression network approach. Sci Rep 10: 12210, 2020.

25. McKhann G, Drachman D, Folstein M, Katzman R, Price D and Stadlan EM: Clinical diagnosis of Alzheimer's disease: Report of the NINCDS-ADRDA Work Group under the auspices of department of health and human services task force on Alzheimer's Disease. Neurology 34: 939-944, 1984.

26. Folstein MF, Folstein SE and McHugh PR: 'Mini-mental state' A practical method for grading the cognitive state of patients for the clinician. J Psychiatr Res 12: 189-198, 1975.

27. Kahle-Wrobleski K, Andrews JS, Belger M, Ye W, Gauthier S, Rentz DM and Galasko D: Dependence levels as interim clinical milestones along the continuum of Alzheimer's Disease: 18-Month results from the GERAS Observational study. J Prev Alzheimers Dis 4: 72-80, 2017.

28. Zeng Q, Zou L, Qian L, Zhou F, Nie H, Yu S, Jiang J, Zhuang A, Wang $\mathrm{C}$ and Zhang H: Expression of microRNA-222 in serum of patients with Alzheimer's disease. Mol Med Rep 16: 5575-5579, 2017.

29. Livak KJ and Schmittgen TD: Analysis of relative gene expression data using real-time quantitative PCR and the 2(-Delta Delta C(T)) Method. Methods 25: 402-408, 2001.

30. Hoo ZH, Candlish J and Teare D: What is an ROC curve? Emerg Med J 34: 357-359, 2017.

31. Gene Ontology Consortium: Gene ontology consortium: Going forward. Nucleic Acids Res 43(Database issue): D1049-D1056, 2015.

32. Kanehisa M, Furumichi M, Tanabe M, Sato Y and Morishima K: KEGG: New perspectives on genomes, pathways, diseases and drugs. Nucleic Acids Res 45: D353-D361, 2017.

33. Dos Santos Picanco LC, Ozela PF, de Fatima de Brito Brito M, Pinheiro AA, Padilha EC, Braga FS, de Paula da Silva CHT, Dos Santos CBR, Rosa JMC and da Silva Hage-Melim LI: Alzheimer's Disease: A review from the pathophysiology to diagnosis, new perspectives for pharmacological treatment. Curr Med Chem 25: 3141-3159, 2018.

34. Van der Kant R, Goldstein LSB and Ossenkoppele R: Amyloid- $\beta$-independent regulators of tau pathology in Alzheimer disease. Nat Rev Neurosci 21: 21-35, 2020.

35. Wei S, Peng W, Mai Y, Li K, Wei W, Hu L, Zhu S, Zhou H, Jie W, Wei Z, et al: Outer membrane vesicles enhance tau phosphorylation and contribute to cognitive impairment. J Cell Physiol 235: 4843-4855, 2020.
36. Bello-Medina PC, González-Franco DA, Vargas-Rodríguez I and Díaz-Cintra S: Oxidative stress, the immune response, synaptic plasticity, and cognition in transgenic models of Alzheimer disease. Neurologia (Engl Ed) S0213-4853(19)30109-4, 2019 (Epub ahead of print)

37. Yoo SM, Park J, Kim SH and Jung YK: Emerging perspectives on mitochondrial dysfunction and inflammation in Alzheimer's disease. BMB Rep 53: 35-46, 2020.

38. Devi L, Prabhu BM, Galati DF, Avadhani NG and Anandatheerthavarada HK: Accumulation of amyloid precursor protein in the mitochondrial import channels of human Alzheimer's disease brain is associated with mitochondrial dysfunction. J Neurosci 26: 9057-9068, 2006.

39. Cheignon $\mathrm{C}$, Tomas M, Bonnefont-Rousselot D, Faller P, Hureau $\mathrm{C}$ and Collin F: Oxidative stress and the amyloid beta peptide in Alzheimer's disease. Redox Biol 14: 450-464, 2018.

40. Simić G, Babić Leko M, Wray S, Harrington C, Delalle I, Jovanov-Milošević N, Bažadona D, Buée L, de Silva R, Di Giovanni G, et al: Tau Protein Hyperphosphorylation and Aggregation in Alzheimer's Disease and other Tauopathies, and possible Neuroprotective Strategies. Biomolecules 6: 6, 2016.

41. Leyns CEG, Ulrich JD, Finn MB, Stewart FR, Koscal LJ, Remolina Serrano J, Robinson GO, Anderson E, Colonna M and Holtzman DM: TREM2 deficiency attenuates neuroinflammation and protects against neurodegeneration in a mouse model of tauopathy. Proc Natl Acad Sci USA 114: 11524-11529, 2017.

42. Langbaum JB, Fleisher AS, Chen K, Ayutyanont N, Lopera F, Quiroz YT, Caselli RJ, Tariot PN and Reiman EM: Ushering in the study and treatment of preclinical Alzheimer disease. Nat Rev Neurol 9: 371-381, 2013.

43. Niemantsverdriet E, Ottoy J, Somers C, De Roeck E, Struyfs H, Soetewey F, Verhaeghe J, Van den Bossche T, Van Mossevelde S, Goeman J, et al: The cerebrospinal fluid A $\beta 1-42 / A \beta 1-40$ ratio improves concordance with Amyloid-PET for diagnosing Alzheimer's Disease in a clinical setting. J Alzheimers Dis 60: 561-576, 2017.

44. Blennow $\mathrm{K}$ and Zetterberg $\mathrm{H}$ : Biomarkers for Alzheimer's disease: Current status and prospects for the future. J Intern Med 284: 643-663, 2018.

45. Cao F, Liu Z and Sun G: Diagnostic value of miR-193a-3p in Alzheimer's disease and miR-193a-3p attenuates amyloid- $\beta$ induced neurotoxicity by targeting PTEN. Exp Gerontol 130: 110814,2020

46. Jia LH and Liu YN: Downregulated serum miR-223 servers as biomarker in Alzheimer's disease. Cell Biochem Funct 34: 233-237, 2016.

47. Ravnskjaer K, Madiraju A and Montminy M: Role of the cAMP pathway in glucose and lipid metabolism. Handb Exp Pharmacol 233: 29-49, 2016.

48. Ricciarelli R and Fedele E: cAMP, cGMP and Amyloid $\beta$ : Three ideal partners for memory formation. Trends Neurosci 41 : 255-266, 2018

49. Nassireslami E, Nikbin P, Payandemehr B, Amini E, Mohammadi M, Vakilzadeh G, Ghadiri T, Noorbakhsh F and Sharifzadeh M: A cAMP analog reverses contextual and tone memory deficits induced by a PKA inhibitor in Pavlovian fear conditioning. Pharmacol Biochem Behav 105: 177-182, 2013.

50. Myeku N, Clelland CL, Emrani S, Kukushkin NV, Yu WH, Goldberg AL and Duff KE: Tau-driven 26S proteasome impairment and cognitive dysfunction can be prevented early in disease by activating cAMP-PKA signaling. Nat Med 22: 46-53, 2016

51. Heras-Sandoval D, Pérez-Rojas JM, Hernández-Damián J and Pedraza-Chaverri J: The role of PI3K/AKT/mTOR pathway in the modulation of autophagy and the clearance of protein aggregates in neurodegeneration. Cell Signal 26: 2694-2701, 2014.

52. Wang $\mathrm{Y}$ and Zhang H: Regulation of autophagy by mTOR signaling pathway. Adv Exp Med Biol 1206: 67-83, 2019.

53. Wang C, Yu JT, Miao D, Wu ZC, Tan MS and Tan L: Targeting the mTOR signaling network for Alzheimer's disease therapy. Mol Neurobiol 49: 120-135, 2014.

54. Hoss AG, Kartha VK, Dong X, Latourelle JC, Dumitriu A, Hadzi TC, Macdonald ME, Gusella JF, Akbarian S, Chen JF, et al: MicroRNAs located in the Hox gene clusters are implicated in huntington's disease pathogenesis. PLoS Genet 10: e1004188, 2014.

55. Miyamoto Y, Mauer AS, Kumar S, Mott JL and Malhi H: Mmu-miR-615-3p regulates lipoapoptosis by inhibiting C/EBP homologous protein. PLoS One 9: e109637, 2014. 
56. Feng H, Gui Q, Wu G, Zhu W, Dong X, Shen M, Fu X, Shi G, Luo H, Yang X, et al: Long noncoding RNA Nespas inhibits apoptosis of epileptiform hippocampal neurons by inhibiting the PI3K/Akt/mTOR pathway. Exp Cell Res 398: 112384, 2021.

57. Wang J, Liu L, Sun Y, Xue Y, Qu J, Pan S, Li H, Qu H, Wang J and Zhang J: MiR-615-3p promotes proliferation and migration and inhibits apoptosis through its potential target CELF2 in gastric cancer. Biomed Pharmacother 101: 406-413, 2018.

58. Maiese K: Forkhead transcription factors: New considerations for alzheimer's disease and dementia. J Transl Sci 2: 241-247, 2016.

59. Santo EE and Paik J: FOXO in neural cells and diseases of the nervous system. Curr Top Dev Biol 127: 105-118, 2018.

60. Maiese K: FoxO proteins in the nervous system. Anal Cell Pathol (Amst) 2015: 569392, 2015.

61. Maiese K: Forkhead transcription factors: Formulating a FOXO target for cognitive loss. Curr Neurovasc Res 14: 415-420, 2017.

62. Shi C, Zhu J, Leng S, Long D and Luo X: Mitochondrial FOXO3a is involved in amyloid $\beta$ peptide-induced mitochondrial dysfunction. J Bioenerg Biomembr 48: 189-196, 2016.

63. Saleem S and Biswas SC: Tribbles pseudokinase 3 induces both apoptosis and autophagy in amyloid- $\beta$-induced neuronal death. J Biol Chem 292: 2571-2585, 2017.

64. Seshacharyulu P, Ponnusamy MP, Haridas D, Jain M, Ganti AK and Batra SK: Targeting the EGFR signaling pathway in cancer therapy. Expert Opin Ther Targets 16: 15-31, 2012.
65. Stupack J, Xiong XP, Jiang LL, Zhang T, Zhou L, Campos A, Ranscht B, Mobley W, Pasquale EB, Xu H and Huang TY: Soluble SORLA enhances neurite outgrowth and regeneration through activation of the EGF Receptor/ERK signaling axis. J Neurosci 40: 5908-5921, 2020.

66. Mansour HM, Fawzy HM, El-Khatib AS and Khattab MM: Potential repositioning of anti-cancer EGFR inhibitors in Alzheimer's Disease: Current perspectives and challenging prospects. Neuroscience 469: 191-196, 2021.

67. Liu F, Lv Q, Du WW, Li H, Yang X, Liu D, Deng Z, Ling W, Zhang Y and Yang BB: Specificity of miR-378a-5p targeting rodent fibronectin. Biochim Biophys Acta 1833: 3272-3285, 2013.

68. Kenny NJ, Sin YW, Hayward A, Paps J, Chu KH and Hui JH: The phylogenetic utility and functional constraint of microRNA flanking sequences. Proc Biol Sci 282: 20142983, 2015.

69. Eun JW, Kim HS, Shen Q, Yang HD, Kim SY, Yoon JH, Park WS, Lee JY and Nam SW: MicroRNA-495-3p functions as a tumor suppressor by regulating multiple epigenetic modifiers in gastric carcinogenesis. J Pathol 244: 107-119, 2018.

This work is licensed under a Creative Commons Attribution-NonCommercial-NoDerivatives 4.0 International (CC BY-NC-ND 4.0) License. 